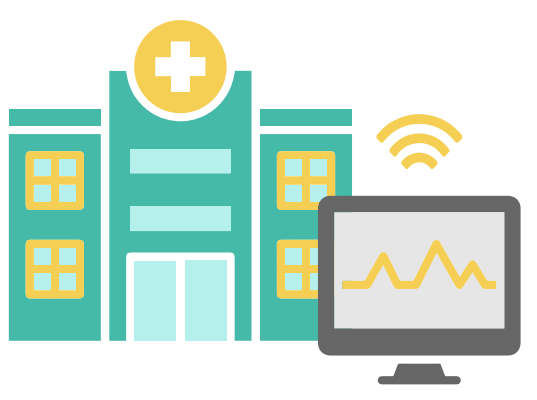

\title{
QUAL O STATUS DA INCORPORAÇÃO DE TECNOLOGIAS EM SAÚDE NO CONTEXTO HOSPITALAR?
}

\author{
WHAT IS THE STATUS OF THE HEALTH TECHNOLOGIES INCORPORATION IN THE \\ HOSPITAL CONTEXT?
}

\begin{abstract}
Rigeldo Augusto Lima
Mestrando do Programa de Pós-Graduação em Gestão e Inovação em Saúde da Universidade Federal do Rio Grande do Norte (UFRN). Especialista em Educação Profissional na Área de Saúde: Enfermagem pela Fundação Oswaldo Cruz (FIOCRUZ/MS). Enfermeiro do Hospital Universitário de Brasília (HUB) da Universidade de Brasília (UnB). E-mail: rigeldo@hotmail.com
\end{abstract}

Joseli Soares Brazorotto

Doutora em Educação Especial pela Universidade Federal de São Carlos (UFSCar). Professora Associada do Departamento de Fonoaudiologia, Universidade Federal do Rio Grande do Norte (UFRN). Docente permanente do Mestrado Profissional em Gestão e Inovação em Saúde (UFRN). Pesquisadora da Base Audição e Linguagem do Laboratório de Inovação Tecnológica em Saúde do Hospital Universitário Onofre Lopes - LAIS/HUOL/UFRN. E-mail: brazorotto@yahoo.com

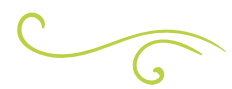

\section{RESUMO}

Introdução: a incorporação de tecnologias em saúde é um tema central nas preocupações de vários países atualmente, em virtude de seu impacto econômico e para a qualidade de vida das populações. Esta pesquisa destaca o fato de que, no Brasil, os hospitais universitários são os principais atores envolvidos na efetiva incorporação de tecnologias para o cuidado em saúde. $\bigcirc$ estudo de todo o processo de incorporação de tecnologias em saúde nessas instituições é, portanto, considerado de extrema importância, por sua relevância para o custo-efetividade dos serviços ofertados aos usuários do SUS. Objetivo: identificar o status da incorporação de tecnologias em saúde, com ênfase nas melhores práticas no contexto hospitalar, considerando as evidências científicas e os documentos nacionais que versam sobre essa temática. Método: consiste em estudo descritivo, analítico e documental, por meio da revisão integrativa da literatura, que se utilizou de três bases de dados (LILACS, PubMed e Periódicos CAPES) no período compreendido entre janeiro de 2014 e maio de 2019 nos idiomas inglês, português e espanhol concomitantemente a uma revisão dos documentos nacionais desde a Lei 8.080/90. Resultados: considerando os 1.307 artigos selecionados para análise, 98\% não abordaram a questão norteadora da presente pesquisa, apresentando escopo clínico. A pesquisa identificou 16 estudos integralmente lidos, entre os quais 05 foram selecionados devido a sua 
especificidade na temática. A partir da revisão documental, consultou um arcabouço teórico-técnico definido e sistematizado sobre como incorporar uma tecnologia com base em normativas legais no sistema de saúde brasileiro. Conclusão: apesar de um arcabouço legal bem delineado, ainda há necessidade de estudos sobre as etapas do processo de incorporação em âmbito hospitalar, fato que representa uma oportunidade para a gestão de custos e da qualidade do serviço ofertado à população brasileira, com forte potencial para a atuação dos hospitais universitários como geradores de inovação nesse cenário.

Palavras-chave: Tecnologias em Saúde. Incorporação. Inovação. Hospital.

\section{ABSTRACT}

Introduction: The incorporation of health technologies is a concern in many countries currently, due it economic and quality of life impacts. In Brazil, university hospitals are the main players involved in the effective incorporation of health care technologies. The study of the whole process of incorporation of health technologies in these institutions is therefore considered of utmost importance because of its relevance to the cost-effectiveness of the services offered to SUS users. Aim: To identify the status of incorporation of health technologies, with emphasis on the best practices of incorporation of these technologies in the hospital context, considering the scientific evidence and national documents on this theme. Method: descriptive, analytical and documentaryresearch, through an integrative literature review, which used three databases (LILACS, PubMed and CAPES) from January 2014 to May 2019 in English, Portuguese and Spanish concurrently with a revision of national documents since Law 8.080/90.

Results: Considering the 1,307 articles selected for analysis, 98\% did not address the guiding question of the present research, presenting a clinical scope. Were identified
16 studies that were fully read, among which 05 were selected due to their specificity in the subject. From the documentary review it was found a defined and systematized theoretical-technical framework on how to incorporate a technology based on legal regulations in the Brazilian health system. Conclusion: Despite a well-designed legal framework, there is still a need for studies on the stages of the hospital incorporation process, a fact that represents an opportunity for cost management and quality of service offered to the Brazilian population, with a strong potential for the University Hospitals as innovation boosters in this scenario.

KEYWORDS: Biomedical Technology. Incorporation. Innovation. Hospitals.

\section{INTRODUÇÃO}

De acordo com a Organização Mundial da Saúde (OMS), tecnologia em saúde é a "aplicação de conhecimentos e habilidades organizados na forma de dispositivos, medicamentos, vacinas, procedimentos e sistemas desenvolvidos para combater um problema de saúde e melhorar a qualidade de vida" (WHO, 2007). Na atualidade, fatores como mais conhecimento sobre o processo saúde-doença, aumento da expectativa de vida da população e aceleração do desenvolvimento tecnológico pressionam a incorporação de tecnologias inovadoras no cuidado em saúde, o que demanda, por sua vez, a elevação dos custos dos sistemas de saúde (LIMA; BRITO; ANDRADE, 2018).

Considera-se, pois, que o processo decisório relativo à incorporação de tecnologias nos diversos sistemas de saúde é influenciado por vários atores, entre eles, as instituições financiadoras, aquelas provedoras dos serviços de saúde, os formuladores de políticas, os profissionais da saúde, os gestores de serviços, os produtores das tecnologias e as associações de pacientes. Todos eles podem exercer papéis 
deliberativos na tomada de decisão, cada um a sua maneira e defendendo os seus interesses, nem sempre explícitos e com julgamentos de valores distintos, os quais podem afetar a incorporação (SANTORO et al., 2018; SANTOS; TRAJMAN; PINTO, 2018; SOUZA; SOUZA, 2018).

Segundo o Ministério da Saúde, o processo de incorporação de novas tecnologias em saúde tem colaborado na escolha de produtos mais adequados, racionalizando gastos e selecionando tecnologias que ofereçam mais benefícios à população. Porém, assim como qualquer mercado importante na economia de um país, a saúde não está imune ao lançamento de tecnologias que não apresentem reais benefícios e que gerem custos desnecessários (BRASIL, 2017b).

Assim, apesar dos avanços alcançados, nosso país deve progredir nesse âmbito. Para tanto, exemplos exitosos de processos de avaliação e incorporação de tecnologias dos sistemas de saúde em países como Austrália, Canadá e Reino Unido poderiam inspirar o estabelecimento sistemático da incorporação de tecnologias nessa realidade. Destaca-se, contudo, que o processo de avaliação e incorporação de tecnologias em saúde no Brasil foi normatizado há menos de dez anos, enquanto, em muitos países, essa discussão ocorre desde a década de 1970, sendo observado que somente no final dos anos 1990 tiveram criadas as suas respectivas agências reguladoras (SANTOS, 2010).

Em um estudo comparativo realizado por Lima, Brito e Andrade (2018), entre as agências governamentais ou independentes que gerenciam o processo de avaliação e incorporação de tecnologias nestes 4 países: Austrália (Medical Service Advisory Committee - MSAC), Canadá (Canadian Agency for Drugs and Technology in Health - CADTH), Reino Unido (National Institute for Health and Clinical Excellence - NICE) e Brasil (Comissão Nacional de Incorporação de Tecnologias no SUS - CONITEC), percebe-se que tal processo não é uniforme e as diferenças identificadas advêm da autoridade na tomada de decisão, do escopo das revisões de evidências, da longevidade/experiência e dos componentes dos processos de incorporação de tecnologias em saúde de cada país. Ainda nessa investigação, os autores destacam que as principais diferenças encontradas nos componentes dos processos de incorporação de tecnologias em saúde entre tais países foram: a possibilidade de apresentação de recursos e prazos para disponibilização das tecnologias após publicação da decisão (existentes no Brasil e Reino Unido) e a avaliação do programa e a seleção de temas por meio de critérios de priorização definidos pelos comitês ou comissões (ausentes somente no Brasil). Outra importante diferença encontrada foi em relação à composição dos comitês. O Brasil foi o único país analisado em que a maioria dos membros da comissão (CONITEC) era representada por servidores de órgãos governamentais.

A atividade de incorporação de tecnologias em saúde em nosso país teve início com a criação, no ano 2000, do Departamento de Ciência e Tecnologia (DECIT) no Ministério da Saúde (MS). No intuito de aprimorar o processo de incorporação no SUS, dando mais agilidade, transparência e eficiência a sua execução, foi criada, então, a Comissão Nacional de Incorporação de Tecnologias no SUS (CONITEC), que passou a ser vinculada à Secretaria de Ciência, Tecnologia e Insumos Estratégicos (SCTIE) do MS, regulamentada pelo Decreto $N^{\circ} 7.646$, de 21 de dezembro de 2011, tendo por objetivo incorporação, exclusão ou alteração de tecnologias pelo SUS, bem como a constituição ou a alteração de protocolos clínicos e diretrizes terapêuticas (BRASIL, 2011c).

Durante o percurso acima e conforme mencionado por Algeri, Costa e Araújo (2018), foram instituídos pelo Ministério da Saúde dois importantes processos no campo da gestão de tecnologias em saúde, a saber: 
- produção, sistematização e difusão de estudos de Avaliação de Tecnologias em Saúde (ATS);

- adoção de um fluxo de incorporação, exclusão ou alteração de novas tecnologias pelo Sistema Único de Saúde (SUS).

Esses processos articulados integram a Política Nacional de Gestão de Tecnologias em Saúde (PNGTS), instituída pela Portaria $N^{\circ} 2.690$ de 05 de novembro de 2009 (BRASIL, 2009b), cujo objetivo foi oferecer condições de equidade, assegurando o acesso da população às tecnologias efetivas e seguras, na tentativa de otimização dos recursos disponíveis. Salienta-se, pois, a importância da sistematização da incorporação de tecnologias em saúde no Brasil, considerando que uma política bem instituída poderá minimizar as consequências do déficit orçamentário e levar o país a ser um modelo também na área de incorporação de tecnologias em saúde, dada a complexidade de nossos contextos no cuidado em saúde e de nossas dimensões continentais.

Os Hospitais Universitários Federais (HUFs), atualmente com contratos vigentes com a Empresa Brasileira de Serviços Hospitalares (Ebserh), deverão naturalmente atuar como polos na incorporação de tecnologias em saúde, dada sua vocação científica e a sua relevância no cenário nacional. Espera-se que, por sua peculiar característica de integrarem-se em uma rede, que os hospitais disponham de um processo de incorporação de tecnologias bem delineado e distinto da ATS e que esse modelo consiga, cumprindo as normativas vigentes, colaborar na diminuição de custos por meio da racionalização do uso das tecnologias em saúde, atendendo, como consequência, as demandas da população (GUIMARÃES, 2014).

Assim posto, a adoção de novos processos e o aprimoramento dos fluxos na avaliação e incorporação de tecnologias em saúde nas instituições hospitalares devem ser pautas constantes, conforme constatado em um estudo realizado por McGregor (2012) com base na avaliação de 15 relatórios para a incorporação de tecnologias em um hospital canadense. Nessa pesquisa, foi observada uma economia global estimada em CAN\$ 9.840.270 (dólares canadenses), valor correspondente a cerca de $\mathrm{R} \$ 28.533 .830,91$ (milhões/reais), de acordo com a taxa de conversão utilizada de 1 Dólar canadense = 2,8997 Real (BRASIL, 2019a) devido à adoção de critérios de incorporação e/ou tomada de decisão bem delineados, tais como: relevância da tecnologia, pontualidade/prazos na tomada de decisão, envolvimento e aceitabilidade por parte dos membros institucionais e transparência nas decisões por meio de publicização. Esses critérios foram considerados como de alto impacto e eram avaliados por um comitê na instituição denominado de Comitê de Política.

Assim, em um cenário no qual a incorporação de tecnologias não é eficiente, mesmo que exista um processo para tal, há perda considerável de recursos financeiros e tecnológicos. Em suma, quando o processo de incorporação de tecnologias em saúde não acontece de maneira sistêmica e não é constantemente avaliado e burilado, há dificuldades para a decisão de se adotar ou não certa tecnologia (por exemplo, por membros de comissões assessoras hospitalares e demais gestores). Os dados do estudo de Ardila, Gómez e Camacho-Cogollo (2016), com pesquisa executada em 25 hospitais terciários da Colômbia, demonstraram que, da amostra selecionada, apenas 68\% das instituições de saúde possuíam um comitê multidisciplinar estruturado como parte do processo de incorporação de tecnologias e apenas $26 \%$ dos profissionais estavam satisfeitos com o processo.

O Brasil não dispõe de investigações sistematizadas sobre a incorporação de tecnologias em contexto hospitalar em todas as regiões do país, mas Queiroz e Barbosa (2003), em estudo realizado em um hospital de referência no estado de São Paulo, constatou que o processo decisório de 
incorporação nesse serviço era caracterizado por fatores subjetivos e a tomada de decisão era realizada frequentemente de modo independente da análise de critérios importantes como o de aplicabilidade da tecnologia e o das vocações estratégicas da instituição. Ademais, em alguns momentos, as decisões eram exclusivas de uma pessoa e/ou categoria profissional, inexistindo, em termos práticos, mecanismos para verificar o impacto e a conveniência da incorporação das tecnologias.

Dada a relevância do emprego de tecnologias em saúde e os avanços inéditos alcançados por processos e produtos para a melhoria da qualidade de serviços de saúde em todo o mundo, urge que se estude o tema da incorporação de tecnologias no âmbito hospitalar e as maneiras de alcançar melhorias nesse processo. Dessa forma, o presente estudo teve por objetivo identificar qual o status da incorporação de tecnologias em saúde no contexto hospitalar, considerando as evidências científicas e os documentos nacionais que versam sobre essa temática, a partir de uma revisão integrativa da literatura e de análise documental nos sites oficiais do governo brasileiro. Espera-se, com este estudo, contribuir para clarificar as virtudes e necessidades do processo de incorporação de tecnologias em saúde no Brasil.

\section{MÉTODOS}

Trata-se de um estudo descritivo, analítico e documental, cuja questão norteadora é: qual o status da incorporação de tecnologias em saúde no âmbito hospitalar? Para a revisão da literatura, utilizou-se o modelo de revisão integrativa proposto por Souza, Silva e Carvalho (2010) que consta de 06 (seis) fases: a) elaboração da pergunta norteadora para um melhor planejamento do estudo; b) busca ou amostragem na literatura; c) coleta de dados; d) análise crítica dos estudos incluídos; e) discussão dos resultados; e f) apresentação da revisão integrativa. As três últimas fases constituem a etapa de análise dos resultados.

$\mathrm{Na}$ etapa de planejamento, foi utilizado um protocolo de revisão, adaptado pelos autores a partir de Mafra e Travessos (2006), considerando também a análise documental, conforme o Quadro 1 a seguir:

\begin{tabular}{|c|c|}
\hline $\begin{array}{l}\text { Formulação da } \\
\text { pergunta }\end{array}$ & $\begin{array}{l}\text { Verificar a literatura e os sites oficiais sobre a incorporação de tecnologias em saúde nos } \\
\text { sistemas de saúde e no ambiente hospitalar. }\end{array}$ \\
\hline Cenário & $\begin{array}{l}\text { Identificar artigos que se relacionam com a formulação da pergunta de pesquisa, devendo } \\
\text { estes se relacionarem ao processo de incorporação de tecnologias em saúde. }\end{array}$ \\
\hline Bases de dados & LILACS, PubMed e Portal de Periódicos CAPES. \\
\hline Palavras-chave & $\begin{array}{l}\text { DeCS: Tecnologias em Saúde, Biomedical Technology, Tecnología Biomédica/ Incorporação, } \\
\text { Incorporation, Incorporación/Inovação, Innovation, Innovación/ Hospital, Hospitals, } \\
\text { Hospitales. }\end{array}$ \\
\hline $\begin{array}{l}\text { Tipos de } \\
\text { Documentos }\end{array}$ & $\begin{array}{l}\text { Selecionar os artigos nas bases de dados elencadas para a revisão integrativa e buscar } \\
\text { complementá-la com a análise documental das principais publicações do Ministério da } \\
\text { Saúde, Presidência da República/Casa Civil e Agência de Vigilância Sanitária (ANVISA). }\end{array}$ \\
\hline Idiomas & Inglês, Português e Espanhol. \\
\hline Critérios de Inclusão & $\begin{array}{l}\text { Incluir artigos científicos, revisados por pares, relevantes à pergunta de pesquisa, que se } \\
\text { adequem à seleção de idiomas e estejam compreendidos entre janeiro de } 2014 \text { e maio } \\
\text { de 2019. No caso dos Periódicos CAPES, houve o refinamento com os seguintes tópicos: } \\
\text { Medicine, Biomedical Materials e Drug Delivery Systems. Para os documentos oficiais, foram } \\
\text { considerados os itens a partir da Lei No } 8.080 \text { de setembro de 1990, portanto, desde a } \\
\text { implementação oficial do Sistema Único de Saúde (SUS). }\end{array}$ \\
\hline Critérios de Exclusão & $\begin{array}{l}\text { Excluir artigos e trabalhos duplicados, com acesso indisponível ao texto na íntegra, artigos } \\
\text { que não respondam à pergunta da pesquisa, outros idiomas que não os selecionados e fora } \\
\text { do período estipulado. }\end{array}$ \\
\hline
\end{tabular}

Quadro 1 - Fase de Planejamento da Revisão Integrativa - Protocolo de revisão.

Fonte: Adaptado de Mafra e Travessos (2006). 
A partir da busca estruturada nas bases de dados selecionadas (segunda etapa do processo de pesquisa), foram utilizadas as seguintes palavras-chaves: Tecnologias em Saúde; Incorporação; Inovação e Hospital, nos 03 idiomas. A seguinte estratégia de pesquisa foi empregada: (tw:(Biomedical Technology)) AND (tw:(Hospitals)) AND (tw:(Innovation)) AND (tw:(Incorporation)); (tw:(Tecnologias em Saúde)) AND (tw:(Incorporação)) AND (tw:(Inovação)) AND (tw:(Hospital)); (tw:(Tecnología Biomédica)) AND (tw:(Hospitales)) AND (tw:(Incorporación)) AND (tw:(Innovación)); (tw:(Biomedical Technology)) AND (tw:(Incorporation)) AND (tw:(Innovation)); (tw:(Tecnologias em Saúde)) AND (tw:(Incorporação)) AND (tw:(Inovação)); (tw:(Tecnología Biomédica)) AND (tw:(Innovación)) AND (tw:(Incorporación)); (tw:(Biomedical Technology)) AND (tw:(Incorporation)); (tw:(Tecnologias em Saúde)) AND (tw:(Incorporação)); (tw:(Tecnología Biomédica)) AND (tw:(Incorporación)). MeSH Terms: biomedical technology; hospitals.
A análise documental foi realizada utilizando-se os sites oficiais dos seguintes órgãos federais: Casa Civil da Presidência da República, Ministério da Saúde (MS) e Agência Nacional de Vigilância Sanitária (ANVISA) com normativas que estivessem relacionadas com a temática da Incorporação de Tecnologias em Saúde a partir da publicação da Lei 8.080 de setembro de 1990 (BRASIL, 1990). No site oficial do Ministério da Saúde, buscou-se refinar a busca tendo como referência o próprio site da CONITEC. Realizou-se, pois, a análise qualitativa dos artigos e documentos oficiais definidos de acordo com os critérios de inclusão para responder à pergunta de pesquisa.

\section{RESULTADOS E DISCUSSÃO}

Obteve-se o seguinte resultado de busca com as associações realizadas, conforme a Tabela 1 abaixo:

Tabela 1 - Resultado da busca nas bases de dados com os descritores selecionados.

\begin{tabular}{|c|c|c|c|c|c|c|}
\hline \multirow[t]{3}{*}{ Descritores } & \multicolumn{6}{|c|}{ Bases de Dados } \\
\hline & \multicolumn{2}{|c|}{ LILACS } & \multicolumn{2}{|c|}{ PubMed } & \multicolumn{2}{|c|}{ Periódicos CAPES } \\
\hline & $\mathbf{E}$ & $\mathbf{S}$ & $\mathbf{E}$ & $\mathbf{S}$ & $\mathbf{E}$ & $\mathbf{S}$ \\
\hline $\begin{array}{l}\text { Biomedical Technology } x \text { Incorporation } \\
\text { x Innovation x Hospitals }\end{array}$ & 1 & 0 & 9 & 0 & 48 & 0 \\
\hline $\begin{array}{l}\text { Tecnologias em Saúde x Incorporação } \\
\text { x Inovação x Hospital }\end{array}$ & 0 & 0 & 0 & 0 & 1 & 0 \\
\hline $\begin{array}{l}\text { Tecnología Biomédica x Incorporación } \\
\text { x Innovación x Hospitales }\end{array}$ & 0 & 0 & 0 & 0 & 1 & 0 \\
\hline $\begin{array}{l}\text { Biomedical Technology } x \\
\text { Incorporation } x \text { Innovation }\end{array}$ & 13 & 2 & 20 & 0 & 41 & 0 \\
\hline $\begin{array}{l}\text { Tecnologias em Saúde x } \\
\text { Incorporação x Inovação }\end{array}$ & 4 & 1 & 0 & 0 & 2 & 0 \\
\hline $\begin{array}{l}\text { Tecnología Biomédica x } \\
\text { Incorporación x Innovación }\end{array}$ & 1 & 1 & 0 & 0 & 1 & 0 \\
\hline Biomedical Technology $x$ Incorporation & 421 & 8 & 459 & 0 & 227 & 0 \\
\hline Tecnologias em Saúde x Incorporação & 29 & 8 & 0 & 0 & 6 & 0 \\
\hline Tecnología Biomédica x Incorporación & 23 & 3 & 0 & 0 & 0 & 0 \\
\hline
\end{tabular}

Legenda: $\mathrm{E}=$ Encontrados; $\mathrm{S}=$ Selecionados

Fonte: Autoria própria (2019).

Ao todo, 1.307 artigos foram encontrados nas bases de dados LILACS, PubMede Periódicos CAPES. Verificou-se que, dos artigos encontrados ( $n=1.307), 04$ artigos (03 em inglês e 01 em 
português) não permitiam acesso ao texto completo e 03 deles foram estudos repetidos (02 em inglês e 01 em espanhol).

Foi realizada a pré-seleção dos textos, baseada na leitura do título e do resumo de todas as referências localizadas, etapa na qual 1.284 estudos foram excluídos. Nessa etapa, $98 \%$ do referencial retornado foram excluídos por não contemplarem o questionamento da pergunta de pesquisa, sendo que os estudos tratavam de aspectos clínicos, com temática específica de nanopartículas, biologia molecular, proteínas e ATS e não especificamente sobre o processo de incorporação. A partir dessa seleção, dos 16 estudos (08 em português, 05 em inglês e 03 em espanhol) lidos na íntegra, 11 foram excluídos, conforme demonstrado na Figura 1 a seguir:

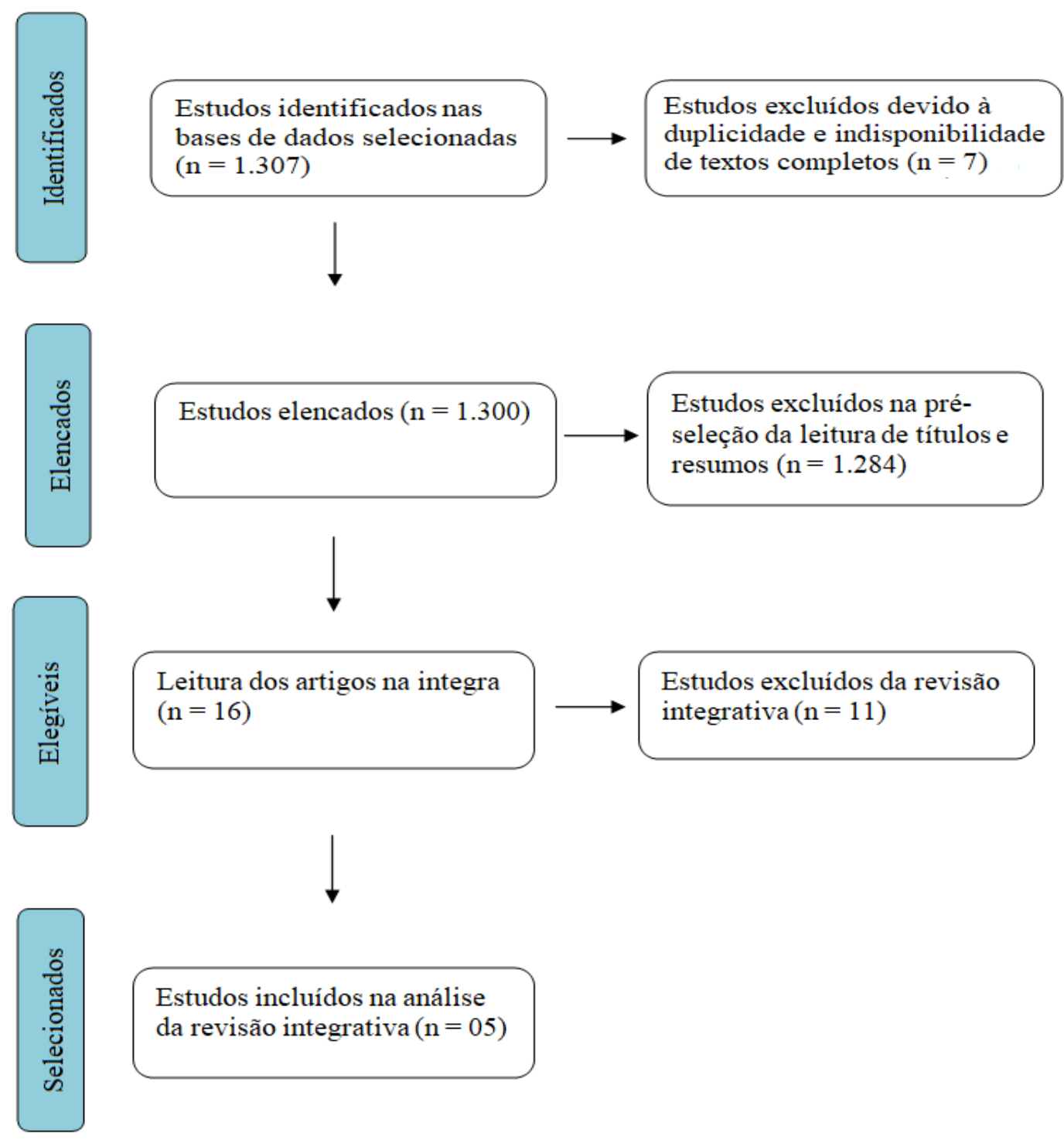

Figura 1 - Fluxograma do processo de seleção dos artigos.

Fonte: Autoria própria (2019).

Ao final, 05 artigos (03 em português, 01 em inglês e 01 em espanhol) foram selecionados para apreciação. Dos 04 trabalhos realizados no Brasil, 01 deles foi publicado internacionalmente. A origem do artigo em espanhol é de pesquisa realizada na Colômbia. A análise dos artigos é descrita no Quadro 2, a seguir: 


\section{Título do artigo}

/ Autor (ano)

Escolhas metodológicas aplicadas aos modelos analíticos e sua influência nos resultados de avaliações econômicas e na tomada de decisão em saúde: um estudo de casos

Santos, Trajman e Pinto (2018)

Incorporação de tecnologias no SUS: as racionalidades do processo de decisão da CONITEC no SUS

Souza e Souza (2018)

Souza e Souza (2018)

Incorporação tecnológica no SUS: o problema e seus desafios

Guimarães, R.

(2014)
Discutir como a escolha de parâmetros e a definição de pressupostos incorporados aos modelos de análise de decisão podem influenciar os resultados e as conclusões das análises de custo-efetividade $e$ custo-utilidade e, por consequência, a alocação de recursos de saúde.

Analisar o processo de decisão da CONITEC referente às recomendações para incorporação ou não de medicamentos.

Discutir o papel da dinâmica tecnológica no aumento dos custos dos sistemas de saúde, sugerindo abordagens como eixos orientadores.
Tipo de estudo: desenho de estudo de caso.

Metodologia/instrumentos: seleção e discussão de três exemplos de avaliações econômicas no Brasil: i) estratégias de vacinação universal infantil contra a hepatite $A$; ii) estratégias diagnósticas para o diagnóstico da infecção latente por tuberculose (ILTB); e iii) uso do brometo de tiotrópio para o tratamento da doença pulmonar obstrutiva crônica (DPOC).

Análise: qualitativa.

Tipo de estudo:analítico-documental.

Metodologia/Instrumentos: análise das decisões tomadas no período de 2010 a 2015. Utilizaram-se como estrategias de produção de dados: avaliação documental treze entrevistas semiestruturadas, envolvendo todos os membros da Conitec, inclusive sua presidente e diretora do DGITS, além de observação não participante das reuniões no período de outubro de 2015 a abril de 2016.

Análise: qualitativa.

Tipo de estudo:

revisão descritiva e analítica sobre várias temáticas especificadas no método.

Metodologia/instrumentos:

revisão descritiva da literatura e análise dos seguintes

temas, a saber: judicialização como uma resultante no debate de cortes; genealogia sumária da ATS e sua linha do tempo no Brasil; a relevância e o impacto da Lei $N^{\circ}$ 12.401/2011, que regulamentou a integralidade no SUS; proposição de desafios para o aprofundamento das ações de ATS com vista à incorporação tecnológica no Brasil; a entrada e o papel da saúde suplementar na incorporação de tecnologias.

Análise: qualitativa
Observou-se que a escolha da estimativa da prevalência da especificidade de um teste e do horizonte temporal gerou resultados de custo-efetividade divergentes.

A literatura sobre falhas e diferenças de métodos que mudem os resultados de estudos de custo-efetividade é escassa. O que motiva as diferentes escolhas pode ser muito diverso; alertamos para o risco das escolhas baseadas em interesses de promover a nova tecnologia.

A análise dos dados evidenciou três tipos de racionalidades presentes no processo de decisão da CONITEC: a racionalidade técnico-sanitária, racionalidade econômica e a racionalidade política. Na prática, a sólida legislação que orienta a avaliação das demandas de incorporação de tecnologias no SUS determinou o predomínio da racionalidade técnico-sanitária.

As abordagens "pragmático-econômica" e "racional defensiva" têm lugar na construção e na execução de políticas de saúde, bem como na litigância jurídica. A institucionalização da ATS em nível global é muito recente. No Brasil, a linha do tempo guarda um atraso relativamente pequeno em relação às experiências dos países centrais.

O processo de incorporação, em particular, o balanço técnico-político que o permeia, sugere uma tese conceitual e operacional que diz respeito a uma necessária separação metodológica, administrativa e física entre as instâncias que, por um lado, priorizam e produzem recomendações e, por outro, as instâncias que, tecnicamente, fazem a ATS.

O padrão de incorporação da saúde suplementar se aproxima do padrão utilizado pelo SUS. 


\begin{tabular}{|c|c|c|c|}
\hline $\begin{array}{l}\text { Título do artigo } \\
\text { / Autor (ano) }\end{array}$ & Objetivo(s) & Métodos & Resultados e Conclusão \\
\hline $\begin{array}{l}\text { Método de incorporación } \\
\text { estratégica de tecnología } \\
\text { biomédica para } \\
\text { instituciones de salud } \\
\text { Ardila, Gómez e Camacho- } \\
\text { Cogollo (2016) }\end{array}$ & $\begin{array}{l}\text { Criar um processo } \\
\text { racional e sistemático } \\
\text { para a incorporação de } \\
\text { equipamentos médicos nas } \\
\text { instituições de saúde. }\end{array}$ & $\begin{array}{l}\text { Tipo de estudo: revisão integrativa e descritivo } \\
\text { transversal. } \\
\text { Metodologia/instrumentos: coleta de informações, } \\
\text { revisão de literatura e implementação de uma pesquisa } \\
\text { para diagnosticar o status dos processos de incorporação } \\
\text { em diferentes instituições de saúde e aprender as } \\
\text { etapas críticas a ser realizadas em cada uma. Para o } \\
\text { desenvolvimento da pesquisa, utilizou-se a ferramenta de } \\
\text { desenho de pesquisa Qualtrics, que gera uma análise dos } \\
\text { resultados em forma gráfica. A pesquisa foi enviada a } 25 \\
\text { engenheiros clínicos (CI) de Instituições de Prestação de } \\
\text { Serviços (IPS) da Colômbia. O método foi implementado } \\
\text { em um caso real com a aquisição de duas tecnologias, } \\
\text { um acelerador linear e um esterilizador a vapor. O } \\
\text { método foi validado com dois especialistas responsáveis } \\
\text { pela aquisição desse equipamento para determinar sua } \\
\text { usabilidade e importância no processo de incorporação } \\
\text { da tecnologia biomédica. } \\
\text { Análises: quantitativa e qualitativa }\end{array}$ & $\begin{array}{l}\text { O método foi validado realizando a incorporação } \\
\text { estratégica de tecnologias biomédicas em uma instituição } \\
\text { de saúde, porque sugere que decisão tomar em cada } \\
\text { etapa do processo. A prova foi a verificação com } \\
\text { especialistas, tendo como feedback validar o método e a } \\
\text { ferramenta. } \\
\text { Os objetivos foram satisfatoriamente cumpridos e } \\
\text { superados ao aplicar o método em um caso real de } \\
\text { análise da incorporação de duas tecnologias biomédicas } \\
\text { na Clínica de Ocidente, na cidade de Cali. }\end{array}$ \\
\hline $\begin{array}{l}\text { Stakeholder value } \\
\text { judgments in decision- } \\
\text { making on the } \\
\text { incorporation, financing } \\
\text { and allocation of new } \\
\text { health technologies in } \\
\text { limited-resource settings: a } \\
\text { potential Brazilian approach } \\
\text { Santoro et al. } \\
\text { (2018) }\end{array}$ & $\begin{array}{l}\text { Analisar os juízos de valor } \\
\text { por trás dos trade-outs de } \\
\text { custo-benefício feitos pelas } \\
\text { partes interessadas na área } \\
\text { da saúde para decidir se } \\
\text { devem ou não incorporar } \\
\text { novas tecnologias em saúde } \\
\text { e como elas devem ser } \\
\text { financiadas e alocadas em } \\
\text { locais de recursos limitados } \\
\text { no Brasil. }\end{array}$ & $\begin{array}{l}\text { Tipo de estudo: exploratório transversal. } \\
\text { Metodologia/instrumentos: De junho de } 2009 \text { a janeiro } \\
\text { de 2010, uma amostra de interessados no setor de saúde } \\
\text { pública e privada foi identificada e convidada a preencher } \\
\text { uma pesquisa on-line com dois questionários: um } \\
\text { coletando informações sociodemográficas/ profissionais e } \\
\text { um capturando preferências de alocação de recursos em } \\
\text { quatro cenários hipotéticos para a incorporação de novas } \\
\text { tecnologias em saúde. } \\
\text { Análises: qualitativa }\end{array}$ & $\begin{array}{l}\text { Um total de } 193 \text { respondentes completaram a pesquisa; } \\
\text { mais da metade eram do sexo masculino (53,9\%) e a } \\
\text { faixa etária mais comum foi de } 31 \text { a } 40 \text { anos (36,8\%). Ao } \\
\text { incorporar novas tecnologias de saúde, isso requer a } \\
\text { redução/eliminação de outros programas de saúde, o } \\
\text { financiamento por meio de impostos ou a escolha de } \\
\text { determinados grupos etários (por exemplo, pessoas } \\
\text { mais jovens versus pessoas idosas), os entrevistados } \\
\text { provavelmente o rejeitarão. Quando se oferece a } \\
\text { opção de limitar o escopo do programa (por exemplo, } \\
\text { prevenção versus tratamento), os entrevistados tendem } \\
\text { a favorecer a prevenção. Pesquisas futuras devem } \\
\text { investigar a perspectiva da sociedade como um todo } \\
\text { para determinar a melhor abordagem para a tomada de } \\
\text { decisão baseada em valores comuns e consenso em um } \\
\text { sistema de saúde particular. }\end{array}$ \\
\hline
\end{tabular}

Quadro 2 - Estudos selecionados para a análise da revisão integrativa.

Fonte: Autoria própria (2019). 
Ao analisar os artigos selecionados, verificou-se que todos se reportaram à temática da pergunta do presente estudo. Os aspectos relacionados aos processos decisórios para auxiliar a aquisição de novas tecnologias foram tratados nos 05 artigos e, destes, 03 eram referentes à tomada de decisão nos sistemas de saúde. Somente 01 artigo tratava da incorporação de tecnologias em saúde no âmbito hospitalar: Ardila, Gómez e Camacho-Cogollo (2016). A temática do custo-efetividade, custo-benefício, custos e financiamento foi abordada em todos os artigos, mesmo que de maneira superficial.

Importante ressaltar que os 11 artigos elegíveis inicialmente, porém não selecionados para a análise final, tratavam exclusivamente do processo de Avaliação de Tecnologias em Saúde (ATS), com relação à vertente econômica, técnico-científica e/ou ética. Outros aspectos abordados nesses artigos se referiam às agências governamentais que realizam a ATS e a estudos sobre como era desenvolvido o processo de trabalho nessas instituições, principalmente $\mathrm{o}$ da CONITEC. Não foi encontrada referência sobre o processo de incorporação ou sobre modelos que auxiliassem na incorporação de tecnologias em saúde mais efetiva no âmbito hospitalar.

Guimarães (2014) reforça que a CONITEC é exemplo de boa governança e accountability (o autor utiliza esse termo pela ausência de tradução direta em português. $O$ significado mais próximo seria a capacidade de prestar contas, de se responsabilizar). Isso se deve a todas as normativas existentes sobre a comissão, principalmente o seu regimento e a transparência de todos os atos, que são publicizadas, o que concorda com a análise realizada no presente estudo.

Ainda segundo Guimarães (2014), para equacionar 0 aspecto técnico e político do processo de incorporação, deve haver uma nítida separação metodológica, técnica, administrativa e física entre as duas instâncias que contemplam a PNGTS: a que realiza a ATS e aquela que prioriza e discute a recomendação para incorporar, ou seja, quem incorpora não avalia e vice-versa. Em suma, o que já acontece ao nível do sistema de saúde brasileiro poderia ser replicado para o âmbito hospitalar.

Souza e Souza (2018), ao analisarem o processo de decisão na incorporação de tecnologias realizada pela CONITEC, identificaram três tipos de racionalidades dos seus membros: a técnico-sanitária, a econômica e a política. Constatou-se que o modelo da racionalidade técnico-sanitária era predominante, devido, principalmente, ao arcabouço normativo legal existente e que o processo de tomada de decisão era considerado coerente para a avaliação das demandas de incorporação no SUS. Porém, deve-se observar que todos os membros da CONITEC são indicados por áreas estratégicas de órgãos da esfera federal, com exceção do CFM, que é uma autarquia e que representa somente uma determinada categoria profissional.

Um ponto importante a ser ressaltado é como ocorre a educação permanente dos membros nesse complexo processo que é a incorporação de tecnologias em um sistema de saúde com características demográfico-epidemiológicas tão heterogêneas em nosso país. A implementação de uma educação permanente para os membros da CONITEC seria um fator importante para a padronização das ações da comissão equalizando possíveis distorções com relação ao seu processo administrativo e dos documentos legais, diminuindo a subjetividade no momento da decisão. Essa é uma característica destacada pelos estudos de Souza e Souza (2018).

Santos, Trajman e Pinto (2018) explicitam que se devem elaborar parâmetros e pressupostos muito bem definidos para a incorporação de tecnologias com relação aos modelos de avaliações econômicas. As análises econômicas ocorrem para comparar os benefícios e os custos de uma determinada tecnologia em relação às já incorporadas anteriormente à tomada de 
decisão. Nesse sentido, a indústria, principalmente a farmacêutica, aproveitando-se dessa lacuna, termina utilizando-se de casos-bases mais favoráveis para tentar incorporar uma tecnologia sem uma análise mais apurada do custo-efetividade e custo-benefício. Os autores ainda fazem uma provocação ao fomentar essa discussão na pauta nacional, para elaborar um padrão de realização de estudos de avaliação econômica que seria um subsídio importante na tomada de decisão na incorporação de tecnologias.

Por sua vez, Santoro et al. (2018) tratam, em seu estudo, da questão do julgamento de valores na incorporação de novas tecnologias. Desse modo, ficou demonstrada a percepção dos diversos participantes da pesquisa em relutar na incorporação de um novo tratamento no SUS quando isso exigia a redução e/ou eliminação de outros programas ou criação de mais taxas ou impostos. Isso pode ser um indicativo para os tomadores de decisões nas devidas instâncias das comissões no âmbito hospitalar. Porém, como os autores ressaltam, há necessidade de se realizarem pesquisas futuras com uma maior amostragem.

Por fim, um dos estudos analisados, de Ardila, Gómez e Camacho-Cogollo (2016), é o único artigo que buscou delinear propriamente um processo de incorporação de tecnologia, de forma padronizada, para a tomada de decisões objetivas, com a existência de comitês de necessidades, de avaliação e de incorporação. Porém, não definiu claramente quais seriam as ferramentas, as (sub)etapas, bem como os atores que participariam de um processo de incorporação efetivo após uma ATS bem realizada e sistematizada.

Em relação a esta pesquisa, conforme descrito na seção método, de modo concomitante à revisão integrativa, executou-se uma revisão documental dos dispositivos legais que regulamentam o processo de incorporação de tecnologias em saúde no Brasil, principalmente aqueles vinculados ao Ministério da Saúde e à Casa
Civil da Presidência da República. Nessa perspectiva, o arcabouço normativo que fundamenta o processo de incorporação de tecnologias em saúde no Brasil está elencado no Quadro 3 a seguir, com os principais dispositivos legais. Destaca-se que, embora alguns deles já tenham sido revogados (estão hachurados em cinza), optou-se por inseri-los na análise considerando que a apresentação em sua cronologia histórica traz melhor compreensão do estado atual. 


\begin{tabular}{|c|c|c|c|c|}
\hline $\begin{array}{l}\text { Leis/Decretos/ } \\
\text { Portarias }\end{array}$ & Data & Dispositivo Legal & Artigos/ Incisos específicos & $\begin{array}{c}\text { Órgão } \\
\text { Responsável }\end{array}$ \\
\hline Lei No 8.080 & 19/09/1990 & $\begin{array}{l}\text { Dispõe sobre as condições para promoção, proteção e recuperação da } \\
\text { saúde, organização e funcionamento dos serviços correspondentes e dá } \\
\text { outras providências. }\end{array}$ & $\begin{array}{l}\text { Artigos 19-M ao 19-U que dispõe sobre a assistência } \\
\text { terapêutica integral e da incorporação de tecnologia } \\
\text { em saúde. }\end{array}$ & $\begin{array}{l}\text { Casa Civil / } \\
\text { Presidência da } \\
\text { República/ }\end{array}$ \\
\hline $\begin{array}{l}\text { Portaria } \\
N^{\circ} 1.418\end{array}$ & 24/07/2003 & $\begin{array}{l}\text { Dispõe sobre a criação do Conselho de Ciência, Tecnologia e Inovação } \\
\text { do Ministério da Saúde. }\end{array}$ & - & $\begin{array}{l}\text { Ministério da } \\
\text { Saúde }\end{array}$ \\
\hline $\begin{array}{l}147^{a} \text { Reunião } \\
\text { do Conselho } \\
\text { Nacional de } \\
\text { Saúde - CNS }\end{array}$ & 07/10/2004 & Política Nacional de Ciência, Tecnologia e Inovação em Saúde (PNCTIS). & 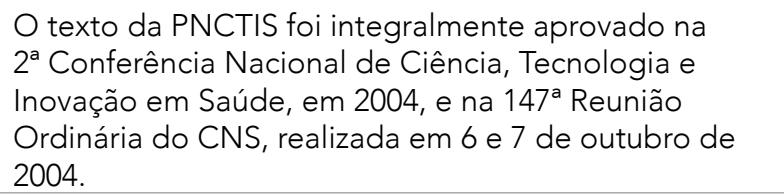 & $\begin{array}{l}\text { CNS / } \\
\text { Ministério da } \\
\text { Saúde }\end{array}$ \\
\hline $\begin{array}{l}\text { Portaria } \\
N^{\circ} 816\end{array}$ & $31 / 05 / 2005$ & $\begin{array}{l}\text { Constitui o Comitê Gestor Nacional de Protocolos de Assistência, } \\
\text { Diretrizes Terapêuticas e Incorporação Tecnológica em Saúde, e dá outras } \\
\text { providências. }\end{array}$ & - & $\begin{array}{l}\text { Ministério da } \\
\text { Saúde }\end{array}$ \\
\hline $\begin{array}{l}\text { Portaria } \\
N^{\circ} 2.510\end{array}$ & 19/12/2005 & $\begin{array}{l}\text { Institui Comissão para Elaboração da Política de Gestão Tecnológica no } \\
\text { âmbito do SUS - CPGT. }\end{array}$ & - & $\begin{array}{l}\text { Ministério da } \\
\text { Saúde }\end{array}$ \\
\hline $\begin{array}{l}\text { Portaria } \\
N^{0} 152\end{array}$ & $19 / 01 / 2006$ & $\begin{array}{l}\text { Institui o fluxo para incorporação de tecnologias no âmbito do Sistema } \\
\text { Único de Saúde. }\end{array}$ & - & $\begin{array}{l}\text { Ministério da } \\
\text { Saúde }\end{array}$ \\
\hline $\begin{array}{l}\text { Portaria } \\
N^{0} 3.323\end{array}$ & $27 / 12 / 2006$ & $\begin{array}{l}\text { Institui a comissão para incorporação de tecnologias no âmbito do } \\
\text { Sistema Único de Saúde e da Saúde Suplementar. }\end{array}$ & - & $\begin{array}{l}\text { Ministério da } \\
\text { Saúde }\end{array}$ \\
\hline $\begin{array}{l}\text { Portaria } \\
\text { Interministerial } \\
N^{0} 2.400\end{array}$ & 02/10/2007 & $\begin{array}{l}\text { Estabelece os requisitos para certificação de unidades hospitalares como } \\
\text { Hospitais de Ensino (HE). }\end{array}$ & $\begin{array}{l}\text { Incisos IV e IX do artigo } 7^{\circ} \text { dispõem sobre o } \\
\text { desenvolvimento de projeto institucional para } \\
\text { avaliação de tecnologias e atividades na padronização } \\
\text { de medicamentos nos HE. }\end{array}$ & $\begin{array}{l}\text { Ministério } \\
\text { da Saúde e } \\
\text { Ministério da } \\
\text { Educação }\end{array}$ \\
\hline $\begin{array}{l}\text { Portaria } \\
N^{\circ} 2.587\end{array}$ & $30 / 10 / 2008$ & $\begin{array}{l}\text { Dispõe sobre a Comissão de Incorporação de Tecnologias do Ministério } \\
\text { da Saúde e vincula sua gestão à Secretaria de Ciência, Tecnologia e } \\
\text { Insumos Estratégicos. }\end{array}$ & - & $\begin{array}{l}\text { Ministério da } \\
\text { Saúde }\end{array}$ \\
\hline $\begin{array}{l}\text { Portaria } \\
N^{\circ} 2.690\end{array}$ & 05/11/2009 & $\begin{array}{l}\text { Institui, no âmbito do Sistema Único de Saúde (SUS), a Política Nacional } \\
\text { de Gestão de Tecnologias em Saúde. }\end{array}$ & - & $\begin{array}{l}\text { Ministério da } \\
\text { Saúde }\end{array}$ \\
\hline Lei $N^{\circ} 12.401$ & 28/04/2011 & $\begin{array}{l}\text { Altera a Lei n } 8.080 \text {, de } 19 \text { de setembro de 1990, para dispor sobre a } \\
\text { assistência terapêutica e a incorporação de tecnologia em saúde no } \\
\text { âmbito do SUS. }\end{array}$ & $\begin{array}{l}\text { Artigo 19-Q cria a Comissão Nacional de Incorporação } \\
\text { de Tecnologias no SUS - CONITEC. }\end{array}$ & $\begin{array}{l}\text { Casa Civil / } \\
\text { Presidência da } \\
\text { República }\end{array}$ \\
\hline $\begin{array}{l}\text { Decreto } \\
N^{0} 7.508\end{array}$ & 28/06/2011 & $\begin{array}{l}\text { Regulamenta a Lei no } 8.080 \text {, de } 19 \text { de setembro de 1990, para dispor } \\
\text { sobre organização do SUS, planejamento da saúde, assistência à saúde e } \\
\text { articulação Inter-federativa, e dá outras providências. }\end{array}$ & $\begin{array}{l}\text { Artigo } 26 \text { que dispõe sobre os protocolos clínicos e as } \\
\text { diretrizes terapêuticas. }\end{array}$ & $\begin{array}{l}\text { Casa Civil / } \\
\text { Presidência da } \\
\text { República }\end{array}$ \\
\hline $\begin{array}{l}\text { Decreto } \\
\text { No } 7.646\end{array}$ & $21 / 12 / 2011$ & $\begin{array}{l}\text { Dispõe sobre a Comissão Nacional de Incorporação de Tecnologias no } \\
\text { SUS e o processo administrativo para incorporação, exclusão e alteração } \\
\text { de tecnologias em saúde pelo SUS, e dá outras providências. }\end{array}$ & - & $\begin{array}{l}\text { Casa Civil / } \\
\text { Presidência da } \\
\text { República }\end{array}$ \\
\hline $\begin{array}{l}\text { Portaria } \\
\text { N}^{\circ} 204\end{array}$ & 07/02/2012 & $\begin{array}{l}\text { Dispõe sobre a Comissão Nacional de Incorporação de Tecnologias no } \\
\text { SUS. }\end{array}$ & - & $\begin{array}{l}\text { Ministério da } \\
\text { Saúde }\end{array}$ \\
\hline
\end{tabular}




\begin{tabular}{|c|c|c|c|c|}
\hline $\begin{array}{l}\text { Leis/Decretos/ } \\
\text { Portarias }\end{array}$ & Data & Dispositivo Legal & Artigos/ Incisos específicos & $\begin{array}{c}\text { Órgão } \\
\text { Responsável }\end{array}$ \\
\hline $\begin{array}{l}\text { Portaria } \\
\mathrm{N}^{0} 2.009\end{array}$ & $13 / 09 / 2012$ & $\begin{array}{l}\text { Aprova o Regimento Interno da Comissão Nacional de Incorporação de } \\
\text { Tecnologias no Sistema Único de Saúde (CONITEC). }\end{array}$ & - & $\begin{array}{l}\text { Ministério da } \\
\text { Saúde }\end{array}$ \\
\hline $\begin{array}{l}\text { Decreto } \\
N^{\circ} 8.077\end{array}$ & $14 / 08 / 2013$ & $\begin{array}{l}\text { Regulamenta as condições para o funcionamento de empresas sujeitas ao } \\
\text { licenciamento sanitário, e registro, controle e monitoramento, no âmbito } \\
\text { da vigilância sanitária, dos produtos de que trata a Lei Nº } 6.360 \text {, de } 23 \text { de } \\
\text { setembro de } 1976 \text {, e dá outras providências. }\end{array}$ & $\begin{array}{l}\text { Artigo } 21 \text { dá possibilidade à CONITEC de solicitar à } \\
\text { ANVISA autorização para uso de medicamentos ou } \\
\text { produtos com indicação distinta daquela aprovada no } \\
\text { registro - uso off label. }\end{array}$ & $\begin{array}{l}\text { Casa Civil / } \\
\text { Presidência da } \\
\text { República }\end{array}$ \\
\hline $\begin{array}{l}\text { Portaria } \\
N^{\circ} 3.390\end{array}$ & $30 / 12 / 2013$ & $\begin{array}{l}\text { Institui a Política Nacional de Atenção Hospitalar (PNHOSP) no âmbito do } \\
\text { SUS, estabelecendo-se as diretrizes para a organização do componente } \\
\text { hospitalar da Rede de Atenção à Saúde (RAS). }\end{array}$ & $\begin{array}{l}\text { Artigo } 23 \text { dispõe que todos os espaços de produção } \\
\text { das ações e serviços de saúde no SUS constituem-se } \\
\text { em campo de prática para ensino, pesquisa e } \\
\text { incorporação tecnológica em saúde [...]. }\end{array}$ & $\begin{array}{l}\text { Ministério da } \\
\text { Saúde }\end{array}$ \\
\hline $\begin{array}{l}\text { Portaria } \\
\text { Interministerial } \\
N^{\circ} 285\end{array}$ & 24/03/2015 & Redefine o Programa de Certificação de Hospitais de Ensino (HE). & $\begin{array}{l}\text { Inciso III do artigo } 4^{\circ} \text {, que trata do estimula à inserção } \\
\text { da instituição na pesquisa, no desenvolvimento e na } \\
\text { gestão de tecnologias em saúde, de acordo com a } \\
\text { necessidade do SUS. }\end{array}$ & $\begin{array}{l}\text { Ministério } \\
\text { da Saúde e } \\
\text { Ministério da } \\
\text { Educação }\end{array}$ \\
\hline $\begin{array}{l}\text { Portaria } \\
\text { No } 26^{\circ} 26\end{array}$ & $12 / 06 / 2015$ & $\begin{array}{l}\text { Aprova os requisitos para submissão e análise de proposta de } \\
\text { incorporação, alteração ou exclusão de tecnologia em saúde no SUS, por } \\
\text { iniciativa do Ministério da Saúde e de Secretarias de Saúde dos Estados, } \\
\text { dos Municípios e do Distrito Federal. }\end{array}$ & - & $\begin{array}{l}\text { Ministério da } \\
\text { Saúde }\end{array}$ \\
\hline $\begin{array}{l}\text { Portaria } \\
N^{\circ} 27\end{array}$ & $12 / 06 / 2015$ & $\begin{array}{l}\text { Aprova o fluxo de trabalho para elaboração e atualização dos Protocolos } \\
\text { Clínicos e Diretrizes Terapêuticas no âmbito da CONITEC no SUS. }\end{array}$ & - & $\begin{array}{l}\text { Ministério da } \\
\text { Saúde }\end{array}$ \\
\hline $\begin{array}{l}\text { Portaria } \\
\text { NN}^{\circ} 30\end{array}$ & 06/01/2017 & $\begin{array}{l}\text { Dispõe sobre a Comissão Nacional de Incorporação de Tecnologias no } \\
\text { SUS e o processo administrativo para incorporação, exclusão e alteração } \\
\text { de tecnologias em saúde pelo SUS. }\end{array}$ & Designar os membros do Plenário da CONITEC. & $\begin{array}{l}\text { Ministério da } \\
\text { Saúde }\end{array}$ \\
\hline $\begin{array}{c}\text { Portaria } \\
N^{\circ} 169\end{array}$ & 04/02/2019 & $\begin{array}{l}\text { Dispõe sobre a Comissão Nacional de Incorporação de Tecnologias no } \\
\text { SUS e o processo administrativo para incorporação, exclusão e alteração } \\
\text { de tecnologias em saúde pelo SUS. }\end{array}$ & Designar os membros do Plenário da CONITEC. & $\begin{array}{l}\text { Ministério da } \\
\text { Saúde }\end{array}$ \\
\hline
\end{tabular}

Quadro 3 - Principais dispositivos legais no processo de incorporação de tecnologias em saúde no Brasil.

Fonte: Autoria própria (2019). 
Percebe-se que, ao analisar o Quadro 3, somente transcorridos 13 anos da Lei $N^{\circ}$ 8.080, de 19 de setembro de 1990 (BRASIL, 1990), que institui o Sistema Único de Saúde (SUS), é que a temática incorporação de tecnologias em saúde passou a ser discutida. Ademais, essas discussões tomaram impulso principalmente pelas ações e normativas implementadas pelo Ministério da Saúde entre os anos de 2004 a 2012, o que culminou com a criação da Comissão Nacional de Incorporação de Tecnologias no SUS (CONITEC), instituída pela Lei No 12.401, de 28 de abril de 2011 (BRASIL, 2011a).

Assim como as agências governamentais de outros países, a CONITEC dispõe de um processo administrativo para incorporação, exclusão e alteração de tecnologias em saúde delineado e suas ações são publicizadas. Não obstante, na documentação analisada, não foi encontrada qualquer forma de especificação ou de perfil mínimo necessário para atuar como membro da CONITEC. Acresça-se, por oportuno que, no intuito de mitigar os contratempos resultantes da eventual substituição de membros ou da mudança na gestão da CONITEC, deveriam ser instituídos procedimentos para assegurar a presença de um número mínimo de membros com mais de 2 anos na comissão, a fim de assegurar a continuidade das atividades, manter a direção do que está sendo executado e o contínuo aprimoramento dos processos. Constata-se também que o arcabouço técnico-legal da comissão com a definição de competências, composição, funcionamento, prazos, consulta pública, estrutura organizacional, todos constantes do regimento interno que também definiu natureza e finalidade, são imprescindíveis para que se tenha um processo de incorporação no SUS mais adequado (LIMA; BRITO; ANDRADE, 2018).

Observou-se ainda que em todas as Portarias Interministeriais MEC/M que dispõem sobre a certificação de Hospital de Ensino (HE), as temáticas sobre ATS, incorporação, atividades de padronização e gestão de tecnologias foram abordadas, pois um dos objetivos da política é estimular a inserção dos Hospitais Universitários na pesquisa, no desenvolvimento e na gestão de tecnologias em saúde, de acordo com as necessidades do SUS. Atualmente, 40 HUFs estão filiados à Empresa Brasileira de Serviços Hospitalares (Ebserh), sendo lócus ideais para o desenvolvimento da política de gestão em tecnologias em saúde.

Sobre a composição da CONITEC, nota-se que esta é exclusivamente representada por órgãos governamentais da esfera federal, com exceção do Conselho Federal de Medicina (CFM). A representatividade atual é definida da seguinte maneira: 08 assentos são de áreas do próprio Ministério da Saúde, 01 da Agência Nacional de Saúde Suplementar (ANS), 01 da Agência Nacional de Vigilância Sanitária (ANVISA), 01 do Conselho Nacional de Secretários de Saúde (CONASS) e finalmente 01 do Conselho Nacional de Secretários Municipais de Saúde (CONASEMS). Deve-se avaliar qual seria a independência das análises de incorporação das tecnologias nesse contexto (LIMA; BRITO; ANDRADE, 2018).

Dessa maneira, observou-se tanto nos registros oficiais como na literatura, que os estudos analisados, assim como as normativas legais vigentes, convergem para um ordenamento na implementação eficiente de uma Avaliação de Tecnologias em Saúde (ATS) que contemple aspectos clínicos, sociais, econômicos e éticos das tecnologias analisadas. Somente a partir disso, os tomadores de decisões das comissões formalmente constituídas em qualquer âmbito poderiam, no momento oportuno da incorporação de tecnologia, com ferramentas e fluxos bem definidos, incorporá-la sem que a subjetividade, a pressão política e dos atores envolvidos no processo pudessem interferir nessa decisão. Reforça-se que, somente a partir de 2011, o Brasil passou a dispor de normativas mais operacionais e estruturantes no processo de incorporação de tecnologias, sendo que a trajetória até 
este momento foi da construção coletiva de uma política em gestão de tecnologias que oferecesse à população equidade no acesso às tecnologias efetivas e seguras na área de saúde.

\section{CONCLUSÃO}

Após revisão da literatura e documental, concluiu-se que: o tema carece de estudos que atestem boas práticas utilizadas na incorporação de tecnologias no âmbito hospitalar. Embora, para o sistema de saúde como um todo, o processo de incorporação de tecnologias em saúde brasileiro seja semelhante ao de países como Austrália, Canadá e Reino Unido, em âmbito hospitalar não se encontraram estudos sobre esse processo. Nesse sentido, um único estudo, realizado na Colômbia, demonstrou a incorporação de tecnologias em saúde no contexto hospitalar com características semelhantes àquelas desenvolvidas nas comissões assessoras das estruturas intra-hospitalares do Brasil. Ademais, a criação da CONITEC representou um marco histórico no processo de incorporação de tecnologias em saúde no Brasil, sendo a sua operacionalização uma forte referência para a incorporação de tecnologias nos hospitais.

Em relação ao arcabouço técnico-legal brasileiro, este é robusto, o que não necessariamente se traduz em uma tomada de decisão técnico-racional adequada, sendo essenciais a qualificação dos atores envolvidos e a independência institucional para a tomada de decisão na incorporação de tecnologias em todos os níveis de saúde. Para que essa incorporação se torne efetiva, há necessidade de aprimoramento no processo de ATS, principalmente com relação à realização de avaliações econômicas, escoIha técnica e uma menor rotatividade dos membros dessas comissões em qualquer nível de gestão. Por conseguinte, novos estudos sobre o processo de incorporação tecnológica no Brasil no contexto hospitalar podem representar uma oportunidade para a gestão de custos e da qualidade do serviço ofertado ao usuário, com forte potencial para a atuação dos Hospitais Universitários como geradores de inovação nesse cenário. 


\section{REFERÊNCIAS}

ALGERI, E. D. B. O.; COSTA, M. C. M. D. R.; ARAÚJO, B. G. A tecnologia como uma ferramenta da qualidade na gestão dos processos de trabalho em saúde na Rede Ebserh. In: GUERRA NETO, C. L. B. et al. (org.). Gestão e inovação em saúde: o que estamos fazendo na Ebserh. Natal: SEDIS-UFRN, 2018. p. 117-140.

ARDILA, M. A.; GÓMEZ, A.; CAMACHO-COGOLLO, J. E. Método de incorporación estratégica de tecnología biomédica para instituciones de salud. Revista Ingeniería Biomédica, Envigado, v. 10, n. 20, p. 35-42, jul./dic. 2016.

BRASIL. Banco Central do Brasil. Conversor de Moedas. Brasília. Disponível em: http://www4.bcb.gov.br/pec/conversao/conversao. asp. Acesso em: 14 jun. 2019a.

BRASIL. Portaria $n^{\circ}$ 169, de 04 de fevereiro de 2019, atualizada em 20 de maio de 2019. Dispõe sobre a Comissão Nacional de Incorporação de Tecnologias no SUS e sobre o processo administrativo para incorporação, exclusão e alteração de tecnologias em saúde pelo SUS. 2019b. Disponível em: http:// conitec.gov.br/images/Legislacao/Portaria_MembroPlenario_ SCTIE_20.05.2019.pdf. Acesso em: 12 jun. 2019.

BRASIL. Portaria $\mathbf{n}^{\circ} \mathbf{3 0}$, de 06 de janeiro de 2017. Dispõe sobre a Comissão Nacional de Incorporação de Tecnologias no SUS e sobre o processo administrativo para incorporação, exclusão e alteração de tecnologias em saúde pelo SUS. 2017a. Disponível em: http:// conitec.gov.br/images/Legislacao/Membros_Portaria30retificada_ DOU_10-01-2017.pdf. Acesso em: 12 jun. 2019.

BRASIL. Secretaria de Ciência, Tecnologia e Insumos Estratégicos. Departamento de Gestão e Incorporação de Tecnologias em Saúde. Diretrizes metodológicas: avaliação de desempenho de tecnologias em saúde. Brasília: Ministério da Saúde, 2017b.

\section{BRASIL. Portaria Interministerial $\mathbf{n}^{\circ} \mathbf{2 8 5}$, de $\mathbf{2 4}$ de março de}

2015. Redefine o Programa de Certificação de Hospitais de Ensino (HE). 2015a. Disponível em: http://bvsms.saude.gov.br/bvs/ saudelegis/gm/2015/prt0285_24_03_2015.html. Acesso em: 10 jun. 2019.

\section{BRASIL. Portaria $\mathbf{n}^{\circ} \mathbf{2 6}$, de $\mathbf{1 2}$ de junho de 2015. Aprova os} requisitos para submissão e análise de proposta de incorporação, alteração ou exclusão de tecnologia em saúde no SUS, por iniciativa do Ministério da Saúde e de Secretarias de Saúde dos Estados, dos Municípios e do Distrito Federal. 2015b. Disponível em: http:// 
conitec.gov.br/images/Legislacao/Portaria26de2015_Requisitos_

SubmissaoeAnalisedeProposta.pdf. Acesso em: 12 jun. 2019.

\section{BRASIL. Portaria $\mathbf{n}^{\circ}$ 27, de 12 de junho de 2015. Aprova $\circ$}

Fluxo de trabalho para elaboração e atualização dos Protocolos Clínicos e Diretrizes Terapêuticas no âmbito da CONITEC no SUS. 2015c. Disponível em: http://conitec.gov.br/images/Legislacao/ Portaria27de2015_FluxoTrabalho_ElaboracaoeAtualizacaoPCDT.pdf. Acesso em: 12 jun. 2019.

\section{BRASIL. Decreto n 8.077, de 14 de agosto de 2013.}

Regulamenta as condições para o funcionamento de empresas sujeitas ao licenciamento sanitário, e o registro, controle e monitoramento, no âmbito da vigilância sanitária, dos produtos de que trata a Lei $n^{\circ} 6.360$, de 23 de setembro de 1976, e dá outras providências. 2013a. Disponível em: http://www.planalto.gov.br/ ccivil_03/_Ato2011-2014/2013/Decreto/D8077.htm. Acesso em: 12 jun. 2019.

BRASIL. Portaria n 3.390, de 30 de dezembro de 2013. Institui a Política Nacional de Atenção Hospitalar (PNHOSP) no âmbito do SUS, estabelecendo- se as diretrizes para a organização do componente hospitalar da Rede de Atenção à Saúde (RAS). 2013b. Disponível em: http://bvsms.saude.gov.br/bvs/saudelegis/gm/2013/ prt3390_30_12_2013.html. Acesso em: 12 jun. 2019.

\section{BRASIL. Portaria $\mathbf{n}^{\circ}$ 204, de 07 de fevereiro de 2012. Dispõe} sobre a Comissão Nacional de Incorporação de Tecnologias no SUS. 2012a. Disponível em: http://bvsms.saude.gov.br/bvs/saudelegis/ gm/2012/prt0204_07_02_2012.html. Acesso em: 12 jun. 2019.

BRASIL. Portaria n².009, de 13 de setembro de 2012. Aprova o Regimento Interno da Comissão Nacional de Incorporação de Tecnologias no Sistema Único de Saúde (CONITEC). 2012b. Disponível em: http://bvsms.saude.gov.br/bvs/saudelegis/gm/2012/ prt2009_13_09_2012.html. Acesso em; 18 jun. 2019.

BRASIL. Lei $\mathbf{n}^{\circ} \mathbf{1 2 . 4 0 1}$, de 28 de abril de 2011. Altera a Lei $n^{\circ}$ 8.080, de 19 de setembro de 1990, para dispor sobre a assistência terapêutica e a incorporação de tecnologia em saúde no âmbito do SUS. 2011a. Disponível em: http://www.planalto.gov.br/ccivil_03/_ Ato2011-2014/2011/Lei/L12401.htm. Acesso em: 12 jun. 2019.

\section{BRASIL. Decreto $\mathbf{n}^{\circ}$ 7.508, de 28 de junho de 2011.}

Regulamenta a Lei n 8.080, de 19 de setembro de 1990, para dispor sobre a organização do SUS, o planejamento da saúde, a assistência à saúde e a articulação Inter federativa, e dá outras providências. 2011b. Disponível em: http://www.planalto.gov.br/ 
ccivil_03/_Ato2011-2014/2011/Decreto/D7508.htm. Acesso em: 12 jun. 2019.

BRASIL. Decreto $\mathbf{n}^{\circ}$ 7.646, de 21 de dezembro de 2011. Dispõe sobre a Comissão Nacional de Incorporação de Tecnologias no SUS e sobre o processo administrativo para incorporação, exclusão e alteração de tecnologias em saúde pelo SUS, e dá outras providências. 2011c. Disponível em: http://www.planalto.gov.br/ CCIVIL_03/_Ato2011-2014/2011/Decreto/D7646.htm. Acesso em: 12 jun. 2019.

BRASIL. Decreto $\mathbf{n}^{\circ}$ 7.082, de 27 de janeiro de 2010. Institui o Programa Nacional de Reestruturação dos Hospitais Universitários Federais - REHUF, dispõe sobre o financiamento compartilhado dos hospitais universitários federais entre as áreas de educação e da saúde e disciplina o regime da pactuação global com esses hospitais. 2010. Disponível em: http://www.planalto.gov.br/ ccivil_03/_Ato2007-2010/2010/Decreto/D7082.htm. Acesso em: 18 jun. 2019.

BRASIL. Secretaria-Executiva. Área de Economia da Saúde e Desenvolvimento. Avaliação de tecnologias em saúde: ferramentas para a gestão do SUS. Brasília: Editora do Ministério da Saúde, 2009a.

BRASIL. Portaria $\mathbf{n}^{\circ}$ 2.690, de 05 de novembro de 2009. Institui, no âmbito do Sistema Único de Saúde (SUS), a Política Nacional de Gestão de Tecnologias em Saúde. 2009b. Disponível em: http:// bvsms.saude.gov.br/bvs/saudelegis/gm/2009/prt2690_05_11_2009. html. Acesso em: 10 jun. 2019.

BRASIL. Portaria $\mathbf{n}^{\circ}$ 2.587, de $\mathbf{3 0}$ de outubro de 2008. Dispõe sobre a Comissão de Incorporação de Tecnologias do Ministério da Saúde e vincula sua gestão à Secretaria de Ciência, Tecnologia e Insumos Estratégicos. 2008. Disponível em: http://conitec.gov.br/ images/Legislacao/Portaria2587_30.10.2008.pdf. Acesso em: 10 jun. 2019.

\section{BRASIL. Portaria Interministerial $\mathbf{n}^{\circ}$ 2.400, de 02 de outubro} de 2007. Estabelece os requisitos para certificação de unidades hospitalares como Hospitais de Ensino. 2007. Disponível em: http:// bvsms.saude.gov.br/bvs/saudelegis/gm/2007/pri2400_02_10_2007. html. Acesso em: 10 jun. 2019.

BRASIL. Portaria $\mathbf{n}^{\circ}$ 3.323, de 27 de dezembro de 2006. Institui a comissão para incorporação de tecnologias no âmbito do Sistema Único de Saúde e da Saúde Suplementar. 2006a. Disponível em: 
http://portalarquivos2.saude.gov.br/images/pdf/2014/janeiro/28/ portaria-CITEC-3323-27dez2006.pdf. Acesso em: 10 jun. 2019.

BRASIL. Portaria $\mathbf{n}^{\circ}$ 152, de 19 de janeiro de 2006. Institui o fluxo para incorporação de tecnologias no âmbito do Sistema Único de Saúde. 2006b. Disponível em: http://bvsms.saude.gov.br/bvs/ saudelegis/gm/2006/prt0152_19_01_2006_comp.html. Acesso em: 10 jun. 2019.

\section{BRASIL. Portaria n 816, de 31 de maio de 2005. Constitui o} Comitê Gestor Nacional de Protocolos de Assistência, Diretrizes Terapêuticas e Incorporação Tecnológica em Saúde, e dá outras providências. 2005a. Disponível em: http://bvsms.saude.gov.br/bvs/ saudelegis/gm/2005/prt0816_31_05_2005.html. Acesso em: 10 jun. 2019.

BRASIL. Portaria $\mathbf{n}^{\circ}$ 2.510, de 19 de dezembro de 2005. Institui Comissão para Elaboração da Política de Gestão Tecnológica no âmbito do SUS - CPGT. 2005b. Disponível em: http://bvsms.saude. gov.br/bvs/saudelegis/gm/2005/prt2510_19_12_2005.html. Acesso em: 10 jun. 2019.

\section{BRASIL. 147 Reunião do CNS, de 07 de outubro de 2004.}

Aprovação da Política Nacional de Ciência, Tecnologia e Inovação em Saúde (PNCTIS) na $2^{a}$ Conferência Nacional de Ciência, Tecnologia e Inovação em Saúde. 2004. Disponível em: https:// conselho.saude.gov.br/recomendacoes/reco_04.htm. Acesso em: 10 jun. 2019.

BRASIL. Portaria n 1.418, de 24 de julho de 2003. Dispõe sobre a criação do Conselho de Ciência, Tecnologia e Inovação do Ministério da Saúde. 2003. Disponível em: http://www.funasa.gov. br/site/wp-content/files_mf/Pm_1418_2003.pdf. Acesso em: 10 jun. 2019.

BRASIL. Lei $\mathbf{n}^{\circ} \mathbf{8 . 0 8 0}$, de 19 de setembro de 1990. Dispõe sobre as condições para a promoção, proteção e recuperação da saúde, a organização e o funcionamento dos serviços correspondentes e dá outras providências. 1990. Disponível em: http://www.planalto.gov. br/ccivil_03/Leis/L8080.htm. Acesso em: 3 jun. 2019.

GUIMARÃES, R. Incorporação tecnológica no SUS: o problema e seus desafios. Ciência \& Saúde Coletiva, v. 19, n. 12, p. 4899-4908, jun. 2014.

LIMA, S. G. G.; BRITO, C.; ANDRADE, C. J. C. O processo de incorporação de tecnologias em saúde no Brasil em uma perspectiva internacional. Ciência \& Saúde Coletiva, jul. 2018. 
Disponível em: http://www.cienciaesaudecoletiva.com.br/ artigos/o-processo-de-incorporacao-de-tecnologias-em-saude-nobrasil-em-uma-perspectiva-internacional/16880?id=16880. Acesso em: 18 jun. 2019.

MAFRA, S. N.; TRAVESSOS, G. H. Estudos primários e secundários apoiando a busca por evidência em engenharia de software, Apêndice I. Rio de Janeiro, 2006. Disponível em: https://www.cin. ufpe.br/ in1037/leitura/EBSE-MafraTravassos-COPPE-2006.pdf. Acesso em: 15 jun. 2019.

\section{McGREGOR, M. The Impact of Reports of the Technology Assessment Unit of the McGill University Health Centre.}

Montreal: Technology Assessment Unit (TAU) of the McGill University Health Centre (MUHC), 2012.

QUEIROZ, A. C.; BARBOSA, A. P. Racionalidade e incorporação de tecnologia em saúde: a experiência de um hospital de alta complexidade em São Paulo. RAE-eletrônica, v. 2, n. 1, jan./jun. 2003.

SANTORO, Luiz et al. Stakeholder value judgments in decisionmaking on the incorporation, financing and allocation of new health technologies in limited-resource settings: a potential Brazilian approach. Rev Panam Salud Publica, Washington, v. 42, p. 1-10, 2018.

SANTOS, M.; TRAJMAN, A.; PINTO, M. Escolhas metodológicas aplicadas aos modelos analíticos e sua influência nos resultados de avaliações econômicas e na tomada de decisão em saúde: um estudo de casos. J Bras Econ. Saúde, São Paulo, v. 10, n. 3, p. 298301, 2018.

\section{SANTOS, V. C. C. As análises econômicas na incorporação de}

tecnologias em saúde: reflexões sobre a experiência brasileira. 2010. 132 f. Dissertação (Mestrado em Saúde Pública) - Escola Nacional de Saúde Pública Sergio Arouca, Fundação Oswaldo Cruz, Rio de Janeiro, 2010.

SOUSA, M. T.; SILVA, M. D.; CARVALHO, R. Revisão integrativa: o que é e como fazer. Faculdade de Enfermagem do Hospital Israelita Albert Einstein - FEHIAE, São Paulo (SP), Einstein, v. 8, 1 Pt 1, p. 102-106, 2010. Disponível em: http://www.scielo.br/pdf/eins/v8n1/ pt_1679-4508-eins-8-1-0102.pdf. Acesso em: 15 jun. 2019. 
SOUZA, K.; SOUZA, L. Incorporação de tecnologias no Sistema

Único de Saúde: as racionalidades do processo de decisão da Comissão Nacional de Incorporação de Tecnologias no Sistema

Único de Saúde. Saúde em Debate, Rio de Janeiro, v. 42, n. esp. 2,

p. 48-60, out. 2018.

WORLD HEALTH ORGANIZATION - WHO. What is a health

technology? Resolution on health Technologies. Geneva:

(WHA60.29), may 2007. 